\title{
Choice of surgical procedures for patients with stage T1 carcinoma of the papilla of Vater: a retrospective study
}

\author{
Zongting Gu ${ }^{1 \wedge}$, Zongze $\mathrm{Li}^{1}$, Wenlong Yu${ }^{2}$, Yongjie Zhang ${ }^{2}$, Chengfeng Wang ${ }^{1}$ \\ ${ }^{1}$ Department of Pancreatic and Gastric Surgery, National Cancer Center/Cancer Hospital, Chinese Academy of Medical Sciences and Peking Union \\ Medical College, Beijing, China; ${ }^{2}$ 2nd Department of Biliary Surgery, Eastern Hepatobiliary Surgery Hospital, Second Military Medical University, \\ Shanghai, China \\ Contributions: (I) Conception and design: Z Gu; (II) Administrative support: Y Zhang, C Wang; (III) Provision of study materials or patients: W Yu, Y \\ Zhang; (IV) Collection and assembly of data: Z Gu, Z Li; (V) Data analysis and interpretation: Z Gu, Z Li; (VI) Manuscript writing: All authors; (VII) \\ Final approval of manuscript: All authors. \\ Correspondence to: Chengfeng Wang, MD. Professor, State Key Lab of Molecular Oncology \& Department of Pancreatic and Gastric Surgery, \\ National Cancer Center/Cancer Hospital, Chinese Academy of Medical Sciences and Peking Union Medical College, Beijing, China. \\ Email: wangchengfeng62@163.com.
}

Background: Given the high incidence of complications after pancreaticoduodenectomy (PD), local resection is being applied to cure stage T1 carcinoma of the papilla of Vater (CPV). In the present study, risk factors related to nodal involvement and prognosis were evaluated so as to enable the choice of optimal surgical procedure for patients with stage T1 CPV.

Methods: A retrospective study of 94 consecutive patients with CPV who underwent PD in our center from 2013 to 2018 was conducted.

Results: A total of 44 patients (46.8\%; 44 of 94) had lymph node metastasis. T1 tumors were subdivided into layer I (the mucosa) and layer II (the submucosa) based on anatomical stratification, and lymph node metastasis did not occur in patients with layer I invasion. The nodal metastasis rate was up to $25 \%$ (6 of 24) in patients with layer II invasion. The gross appearance, depth of duodenal invasion, pT stage and perineural invasion were risk factors related to nodal involvement. Only the depth of duodenal invasion remained a significant independent factor $(\mathrm{P}=0.003)$. Multivariate Cox analysis indicated that depth of duodenal invasion $(\mathrm{P}=0.001)$, nodal involvement $(\mathrm{P}<0.001)$, and venous invasion $(\mathrm{P}<0.001)$ were independent prognostic factors. The depth of duodenal invasion is the only independent risk factor related to nodal involvement and prognosis.

Conclusions: The optimal surgical option should be PD with radical lymphadenectomy for patients with stage T1 CPV; only patients with duodenal invasion limited to the mucosa are suitable for local resection. A modified $\mathrm{T}$ category needs to be proposed based on the detailed depth of duodenal invasion.

Keywords: Carcinoma of the papilla of Vater (CPV); ampullary tumor; lymph node metastasis; pancreatoduodenectomy; local resection; prognostic factor

Submitted Apr 24, 2020. Accepted for publication Sep 17, 2020.

doi: $10.21037 /$ tcr-20-1914

View this article at: http://dx.doi.org/10.21037/tcr-20-1914

$\wedge$ ORCID: 0000-0002-7800-7988. 


\section{Introduction}

Carcinoma of the papilla of Vater (CPV) accounts for about $0.6 \%$ of all gastrointestinal carcinomas, so it is a very rare tumor (1). Nodal involvement is the main pattern of tumor spread and is known as one of most important factors related to poor prognosis in CPV (2-5). Therefore, lymphadenectomy play an important role in radical resection. CPV tends to be detected in patients in the relatively early stage, and these patients have a better prognosis and higher resection rate than those with periampullary tumors $(4,6,7)$. Thus, radical pancreatoduodenectomy (PD) has been considered the curative treatment of choice $(7,8)$. Currently, the mortality of $\mathrm{PD}$ is less than $5 \%$, and the long-term survival is satisfactory; however, the high incidence of complications associated with PD is still not widely accepted. Alternative and less-invasive approaches are imperative. Some studies have shown that papillary local resection including endoscopic or transduodenal papillectomy with minimal invasiveness is potentially curative in patients with stage T1 CPV (9-11). In stage T1 CPV, lymph node metastasis seems to be the most significant prognostic factor because vascular invasion or distant metastasis is extremely rare in such patients $(2,4,11)$. Unfortunately, local resection does not involve lymph node dissection, potential nodal involvement increases the chance of tumor recurrence in stage T1 CPV. Therefore, the absence of lymph node metastasis is the key to apply this technique. Otherwise, PD with lymphadenectomy is a reasonable choice. Currently, an accurate preoperative assessment of lymph node status is hard to achieve, which can only be obtained by careful pathological examination after complete nodal clearance. The identification of clinicopathologic factors associated with lymph node metastasis may provide alternative indirect approach to identify lymph node status and offer some surgical guidelines for patients suitable. Risk factors related to nodal involvement have rarely been mentioned in previous studies. Some reports have indicated that the presence of duodenal invasion is a risk factor related to nodal involvement in CPV $(10,12)$. However, the relationship between the detailed depth of duodenal invasion and lymph node metastasis still needs to be clarified.

Because of the low incidence of CPV, the previous studies that have focused on prognostic factors in CPV had small sample sizes and varying conclusions $(3-5,12,13)$. The prognostic factors for $\mathrm{CPV}$ remain controversial. Analysis of prognostic factors after radical resection may provide a guide for selected patients to choose appropriate surgical procedure or adjuvant treatment, though data supporting the role of adjuvant therapies are limited (14). Advanced AJCC T stage and duodenal invasion are known as strong factors for predicting poor prognosis in CPV. Moreover, the impact of duodenal invasion depth on prognosis is receiving increasing attention $(6,15)$. The $8^{\text {th }}$ edition of the AJCC staging system updates the new definition of the $\mathrm{T}$ stage based on the depth of duodenal invasion (16). However, the impact of new $\mathrm{T}$ staging and detailed depth of duodenal invasion on prognosis remains unclear.

Given these considerations, this retrospective study aims to evaluate risk factors related to lymph node metastasis and prognosis so as to enable the choice of optimal surgical procedure for patients with stage T1 CPV. We present the following article in accordance with the STROBE reporting checklist (available at http://dx.doi.org/10.21037/tcr-201914).

\section{Methods}

\section{Patient characteristics}

A retrospective study of adult patients with CPV who underwent PD (Whipple procedure) and regional lymphadenectomy at Eastern Hepatobiliary Surgery Hospital (Shanghai, China) from Jan. 2013 to Dec. 2018 was conducted. The study was conducted in accordance with the Declaration of Helsinki (as revised in 2013). The study was reviewed and approved by the institutional review board of Eastern Hepatobiliary Surgery Hospital (EHBHKY2019-003-038), Shanghai, China. Written informed consent was obtained from all patients. All cases of CPV were found and confirmed by postoperative pathology. Patients with tumors extensively involving the bile duct and pancreas, patients with difficulty identifying the tumor origin, patients with ambiguous or incomplete pathological results, and patients with adenoma and hyperplasia were excluded. Ninety-four cases were obtained in the study, including 51 males and 43 females with a mean age of 53.6 years (34-78 years). The reviewed preoperative data included patient demographic characteristics (age and sex), preoperative examination results (tumor markers, physical examinations, endoscopy, and forceps biopsy), surgical data (type of resection, lymph node status, pancreatic invasion, perineural invasion and venous invasion), pathological characteristics (gross appearance, tumor size, depth of 
duodenal invasion, differentiation, and $\mathrm{pT}$ staging), and postoperative data (recurrence status, date of death, and follow-up deadline).

\section{Operative procedures and extent of regional lymphadenectomy}

Conventional PD and pylorus-preserving pancreaticoduodenectomy (PPPD) were performed in 84 and 10 patients, respectively. We performed regional nodal clearance in all patients. The extent of lymph node dissection covers the lymphatic, neural, and soft tissues in the posterior or anterior pancreaticoduodenal, hepatoduodenal ligament, right celiac, right side of superior mesenteric artery and paraaortic area. The key points of standard PD operation have been previously reported (7).

\section{Postoperative pathology}

Histological examination of specimens was performed to identify the tumor size, gross type, histological grade, depth of duodenal invasion, pancreatic invasion, nodal involvement, venous invasion, and perineural invasion. The extent of the primary tumor was classified into four pT stages based on the new AJCC Cancer Staging $\left(8^{\text {th }}\right.$ edition) (16): pT1, tumors invading within or beyond the sphincter of Oddi or into the submucosa; pT2, tumors invading into the muscularis propria; $\mathrm{pT} 3$, tumors invading the pancreas or extending into peripancreatic tissue; and pT4, tumors involving the celiac axis, superior mesenteric artery, or common hepatic artery. The macroscopic classifications were divided into two types based on their predominant shape (17): ulcer type and non-ulcer type. The depth of duodenal invasion was categorized into four layers based on anatomical structure $(18,19)$ : layer I, invasion limited to the mucosa; layer II, invasion of the submucosa (beyond the mucosa but not reaching the muscularis propria, including the sphincter of Oddi); layer III, invasion of the muscularis propria; and layer IV, invasion of the serosa or beyond (pancreatic invasion or adjacent tissue). The histological grade was divided into three categories based on the predominant features (17), consisting of well differentiated (G1), moderately differentiated (G2), and poorly differentiated (G3). Lymph node status was divided into two levels (17): $\mathrm{N}_{0}$, no regional lymph node metastasis; and $\mathrm{N}_{1}$, regional lymph node metastasis. Lymph node metastasis was identified on routine histological examination by hematoxylin and eosin. Two pathologists separately evaluated the histopathological features of all cases after operation.

\section{Follow-up}

Patients were advised to regularly undergo computed tomography (CT) every 6 months for 5 years after surgery. Follow-up data on long-term survival were obtained through direct phone contact, correspondence, and review of death registration records. The follow-up deadline was December 2019. The median follow-up time was 25 months (mean 36 months). Survival time was defined as months between the operation day and the follow-up deadline or date of death.

\section{Statistical analysis}

Risk factors related to nodal involvement were analyzed by univariate statistical analysis via the Chi-squared test or Fisher's exact test, when applicable. Cumulative survival curves were obtained using the Kaplan-Meier method and survival difference of each factor was compared using logrank tests. ROC tests were used to assess the prediction accuracy of the model. Logistic regression was used to confirm the independent factors related to lymphatic metastasis using a stepwise approach. Multivariate analysis of factors affecting survival was based on the Cox proportional hazards model. A $\mathrm{P}$ value of less than 0.05 was considered statistically significant. Data analysis was performed using IBM SPSS Statistics software Version 21.0 for Windows (SPSS, Inc., Chicago, IL, USA).

\section{Results}

\section{Clinicopathological features}

PD and PPPD with radical regional lymphadenectomy were performed in 84 and 10 patients, respectively. The mean tumor size was $2.2 \mathrm{~cm}$ (range, $0.5-4.5 \mathrm{~cm}$ ). Histologically, ulcer-type and non-ulcer-type tumors were found in 21 and 73 patients, respectively. All patients were confirmed to have adenocarcinoma. Pathologically, duodenal invasion of layers I, II, III and IV was identified in 6, 24, 20 and 44 patients, respectively. Similarly, T1, T2, T3 and T4 tumors were identified in $30,20,35$ and 9 patients, respectively. Specimens also confirmed pancreatic, venous, and perineural invasion in 10,4 , and 11 patients, respectively. 


\section{Nodal involvement analysis}

\section{Frequency of lymph node metastasis}

The number of removed nodes ranged from 5 to 61 (median, 16.5). A total of 44 patients $(46.8 \%$; 44 of 94) had nodal involvement with a median number of two positive nodes (range, 1-10), while 50 patients were negative. In the posterior or anterior pancreaticoduodenal, hepatoduodenal ligament, right celiac and right side of superior mesenteric artery, rates of nodal involvement were 34\% (32/94), 12\% (11/94), 7\% (7/94) and 22\% (21/94), respectively. Paraaortic nodes had no metastases. Regarding gross appearance, ulcer-type lesion $(66.7 \% ; 14$ of 21$)$ was more likely to have lymph node metastasis than non-ulcer-type $(41.1 \% ; 30$ of 73). The depth of duodenal invasion was significantly deeper in node-positive patients than in the negative ones, and the incidence of lymph node involvement significantly increased, along with deepening duodenal invasion. Lymph node metastasis did not occur in patients with layer I invasion. The rate of lymph node metastasis was up to $25 \%$ (6 of 24 ) in patients with layer II invasion, $50 \%$ (10 of 20) in patients with layer III invasion, and 63.6\% (28 of 44) in patients with layer IV invasion. Likewise, lymph node positivity increased with increasing $\mathrm{pT}$ stage. The nodal metastasis rates of $\mathrm{pT} 1, \mathrm{pT} 2, \mathrm{pT} 3$, and $\mathrm{pT} 4$ tumors were $20 \%$ (6 of 30 ), $50 \%$ (10 of 20 ), $60 \%$ (21 of 35 ), and $77.8 \%$ (7 of 9), respectively. In addition, all patients with perineural invasion were confirmed to have positive lymph nodes.

\section{Clinicopathological factors associated with lymph node metastasis}

The Chi-squared analysis revealed that the gross appearance $(\mathrm{P}=0.038)$, depth of duodenal invasion $(\mathrm{P}=0.001)$, pT stage $(\mathrm{P}=0.001)$ and perineural invasion $(\mathrm{P}<0.001)$ were significantly related to lymph node metastasis (Table 1). Other factors including tumor size, histology, pancreatic invasion, and venous invasion were not related to nodal involvement. A logistic regression analysis was subsequently performed to determine the most significant variables, which indicated that only depth of duodenal invasion remained significantly and independently related to lymph node metastasis $(\mathrm{P}=0.003)$ (Table 1). pT stage was not included because this factor was partly overlapped with duodenal invasion.

\section{Survival analysis}

There was no 30 -day mortality. A total of 8 patients $(8.5 \%$,
8/94) were lost to follow-up. The 1-, 3-, and 5-year survival rates for the 94 patients were $81.7 \%, 55.6 \%$, and $45.2 \%$, respectively (Figure 1). The median survival time was 44.3 months. Univariate log-rank analysis indicated that gross appearance $(\mathrm{P}=0.016)$, depth of duodenal invasion $(\mathrm{P}<0.001)$, p T staging $(\mathrm{P}<0.001)$, nodal involvement $(\mathrm{P}<0.001)$, pancreatic invasion $(\mathrm{P}=0.004)$, venous invasion $(\mathrm{P}<0.001)$ and perineural invasion $(\mathrm{P}<0.001)$ were the significant prognostic factors (Table 2). Figures 2 and 3 show Kaplan-Meier survival curves as related to $\mathrm{pT}$ stage and depth of duodenal invasion, respectively. ROC test showed that the prediction accuracy of model based on duodenal invasion (AUC $=0.72$ ) was better than the one based on $\mathrm{pT}$ stage (AUC $=0.69)$ (Figure 4). Multivariate Cox regression analysis indicated that depth of duodenal invasion $(\mathrm{P}=0.001)$, nodal involvement $(\mathrm{P}<0.001)$, and venous invasion $(\mathrm{P}<0.001)$ were independent risk factors related to poor prognosis (Table 2). pT stage was not included because this factor was partly overlapped with duodenal invasion.

\section{Discussion}

Our study confirms that lymph node metastasis is one of the strongest prognostic factors in CPV. Previously, there have rarely been studies that have focused on lymph node metastasis in CPV, and this study is an effective attempt to reveal the detailed relationship between clinicopathological factors and nodal involvement. Moriya et al. (4) reported that gross appearance and histological differentiation were related to lymph node metastasis in CPV. The correlation between tumor size and lymph node metastasis is still controversial $(2,10)$. Aiura et al. (10) reported that the presence of duodenal infiltration and perineural invasion were risk factors for nodal spread. Kayahara et al. (12) divided ampullary invasion into three degrees, and they concluded that the depth of tumor invasion was related to lymph node metastasis. In the current study, our results are consistent with these reports (Table 1); more importantly, we stratified duodenal invasion in greater detail and indicated that the detailed depth of duodenal invasion and $\mathrm{T}$ staging were associated with nodal involvement in CPV. Moreover, the depth of duodenal invasion was confirmed to be the strongest risk factor related to lymph node metastasis. The pT stage was excluded because of its partial overlap with duodenal invasion. Unfortunately, our study showed no relationship with nodal involvement in tumor size and differentiation, although large tumors are not well suited for local resection because of the technical difficulties 
Table 1 Univariate and multivariate analyses of the risk factors for lymph node metastasis in patients with carcinoma of the papilla of Vater after surgery

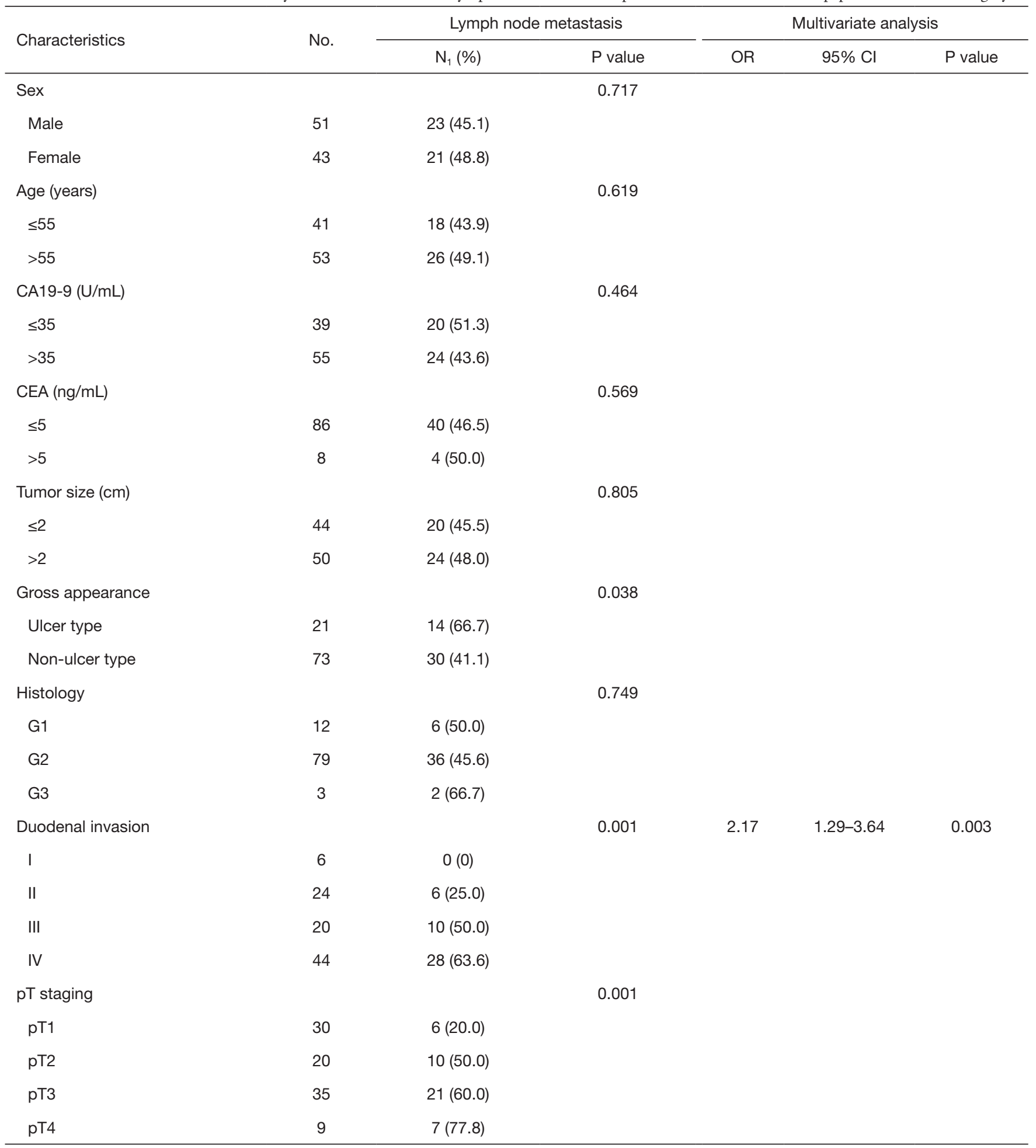

Table 1 (continued) 
Table 1 (continued)

\begin{tabular}{|c|c|c|c|c|c|c|}
\hline Characteristics & No. & \multicolumn{2}{|c|}{ Lymph node metastasis } & \multicolumn{3}{|c|}{ Multivariate analysis } \\
\hline Pancreatic invasion & & & 0.181 & & & \\
\hline Present & 10 & $7(70.0)$ & & & & \\
\hline Absent & 84 & $37(44.0)$ & & & & \\
\hline Present & 4 & $2(50.0)$ & & & & \\
\hline Absent & 90 & $42(46.7)$ & & & & \\
\hline Perineural invasion & & & $<0.001$ & & & \\
\hline Present & 11 & $11(100.0)$ & & & & \\
\hline
\end{tabular}

$\mathrm{Cl}$, confidence interval; OR, odds ratio; significant at the level of $\mathrm{P}<0.05$. G1, well-differentiated type; G2, moderately differentiated type; G3, poorly differentiated type; I, invasion limited to the sphincter of Oddi; II, invasion of the submucosa; III, invasion of the muscularis propria; IV, invasion of the serosa or beyond.

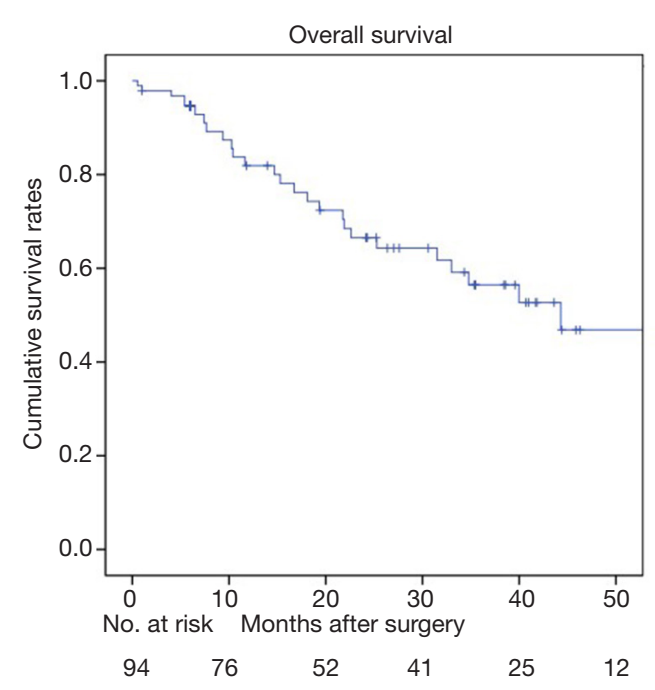

Figure 1 Kaplan-Meier overall survival curve of the entire cohort of 94 patients who underwent resection for carcinoma of the papilla of Vater (median survival was not reached).

associated with ensuring tumor-free margins.

Pathologically, in ulcer lesions of CPV, the tumor destroys the basement membrane of the duodenal wall, and transfers through lymphatic vessels within the mucosa and submucosa to lymph nodes. Therefore, it is reasonable that ulcerative tumors are more likely to have lymph node metastasis (66.7\% vs. $41.1 \%)$. Meanwhile, as tumor invasion deepens, cancer cells will get access to the larger lymphatic vessels within the submucosa more likely. Thus, the deeper the tumor invades, the greater the possibility of nodal involvement. In the current study, the incidence of nodal positivity increased with deepening of the depth of invasion ( 0 in I, $25 \%$ in II, $50 \%$ in III, and $63.6 \%$ in IV), as well as with increases in the pT stage $\left(20 \%\right.$ in $\mathrm{pT}_{1}, 50 \%$ in $\mathrm{pT}_{2}$, $60 \%$ in $\mathrm{pT}_{3}$, and $77.8 \%$ in $\mathrm{pT}_{4}$ ). In addition, thin-walled lymphatic vessels which are widely distributed within nerve plexuses around the pancreas and superior mesenteric artery are considered to be important pathways for perineural infiltration (20), in agreement with our data that all patients with perineural invasion had nodal involvement.

In CPV, the incidence of lymph node metastasis has been reported to range from $41.4 \%$ to $50 \%(4,12,21)$. In the present study, the rate of nodal spread was $46.8 \%$. An accurate preoperative evaluation of nodal status is a priority for local resection. Currently, transabdominal ultrasonography (US), CT, magnetic resonance imaging (MRI), and endoscopic ultrasonography (EUS) are the major preoperative modalities for evaluating nodal status. However, the direct diagnostic accuracy of nodal involvement is not optimistic. The diagnostic accuracy is 7\% in US, $33 \%$ in CT/MR, and $47 \%$ in EUS (22), which can only provide suspicious features. Given the difficulties in accurate preoperative diagnosis of lymph node metastasis, our research offers another possible indirect approach. Among the risk factors related to lymph node metastasis, pT staging and perineural invasion can be acquired only 
Table 2 Univariate and multivariate analyses of prognostic factors in patients with carcinoma of the papilla of Vater after surgery

\begin{tabular}{|c|c|c|c|c|c|c|c|}
\hline Characteristics & No. & \multicolumn{3}{|c|}{ Univariate analysis } & \multicolumn{3}{|c|}{ Multivariate analysis } \\
\hline Sex & & & $0.39-1.85$ & 0.695 & & & \\
\hline Male & 51 & 74 & & & & & \\
\hline$\leq 55$ & 41 & 44.2 & & & & & \\
\hline$>55$ & 53 & 72 & & & & & \\
\hline CA19-9 (U/mL) & & & $0.43-2.06$ & 0.888 & & & \\
\hline$\leq 5$ & 86 & 44.2 & & & & & \\
\hline$>5$ & 8 & 44 & & & & & \\
\hline Tumor size (cm) & & & $0.54-2.58$ & 0.674 & & & \\
\hline$\leq 2$ & 44 & 44.2 & & & & & \\
\hline$>2$ & 50 & 59 & & & & & \\
\hline Gross appearance & & & $0.17-0.86$ & 0.016 & & & \\
\hline Ulcer type & 21 & 31.1 & & & & & \\
\hline Duodenal invasion & & & $1.93-6.03$ & $<0.001$ & 3.39 & $1.68-6.84$ & 0.001 \\
\hline I & 6 & 74 & & & & & \\
\hline II & 24 & 59 & & & & & \\
\hline III & 20 & 39.2 & & & & & \\
\hline IV & 44 & 14.8 & & & & & \\
\hline pT staging & & & $2.22-6.41$ & $<0.001$ & & & \\
\hline pT1 & 30 & 74 & & & & & \\
\hline рT2 & 20 & 39.2 & & & & & \\
\hline pT3 & 35 & 19 & & & & & \\
\hline pT4 & 9 & 7.5 & & & & & \\
\hline
\end{tabular}

Table 2 (continued) 
Table 2 (continued)

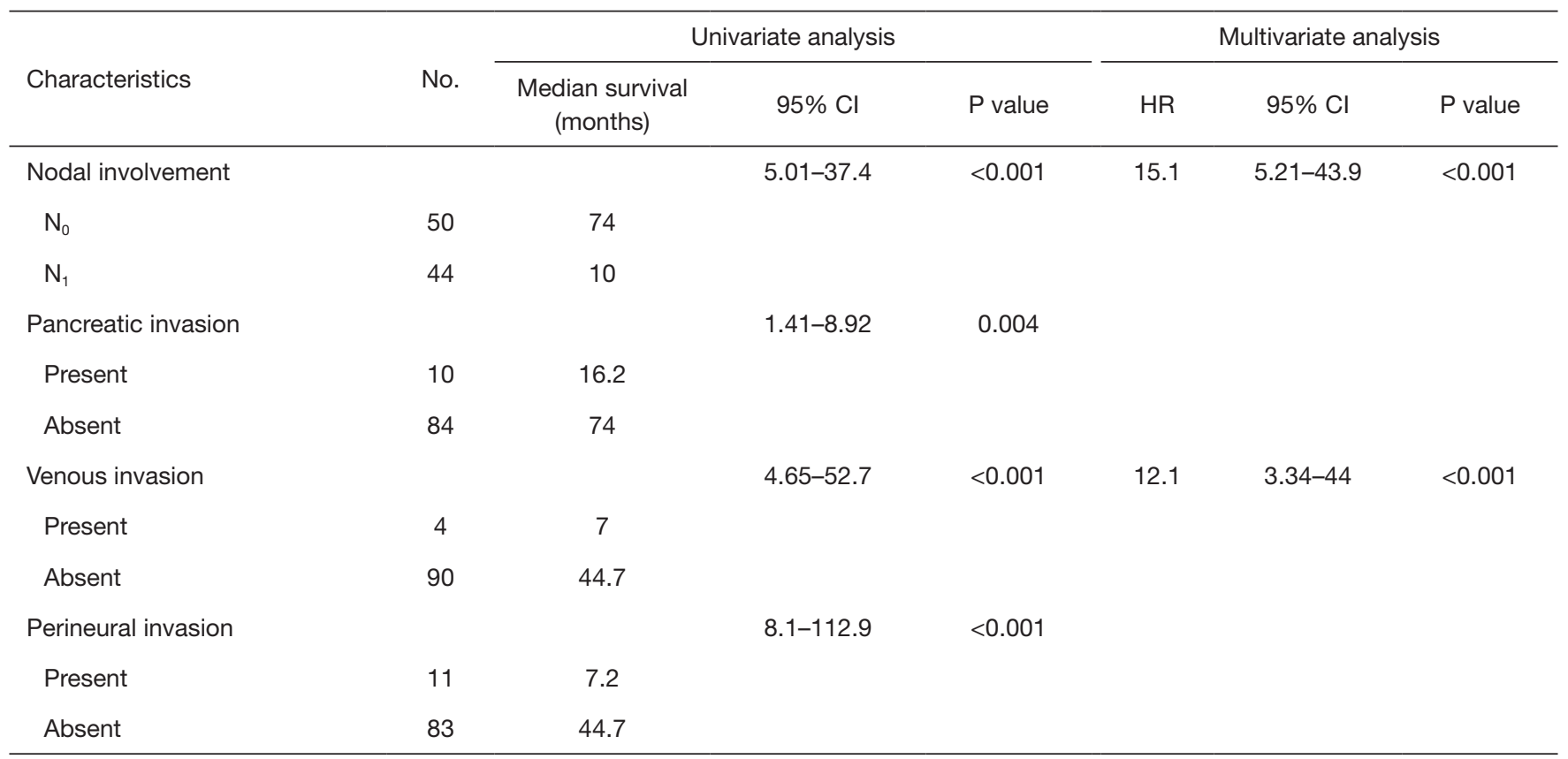

$\mathrm{Cl}$, confidence interval; HR, hazard ratio; significant at the level of $\mathrm{P}<0.05$. G1, well-differentiated type; G2, moderately differentiated type; G3, poorly differentiated type; I, invasion limited to the sphincter of Oddi; II, invasion of the submucosa; III, invasion of the muscularis propria; IV, invasion of the serosa or beyond.

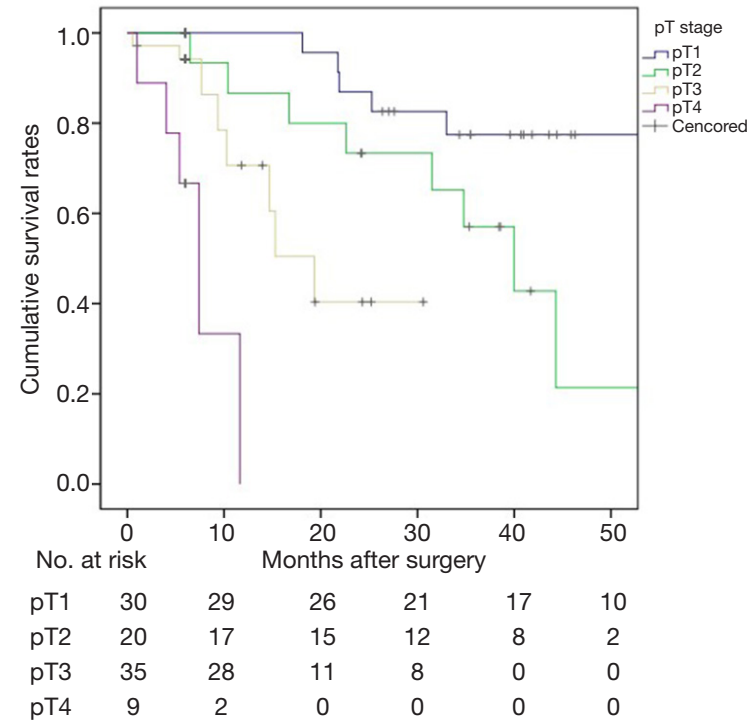

Figure 2 Kaplan-Meier survival curves for patients with pT stages (AJCC, 8th edition) after resection for carcinoma of the papilla of Vater. There was a clinically important difference in survival among the groups (log-rank test, $\mathrm{P}<0.001$ ). However, $\mathrm{T} 2$ and $\mathrm{T} 3$ curves were not independent of each other in the figure $(\mathrm{P}=0.073)$. from postoperative pathology; however, gross appearance and depth of duodenal invasion can be preoperatively observed by noninvasive means [endoscopy, EUS, intraductal ultrasonography (IDUS)]. Multivariate analysis indicated that the depth of duodenal invasion was the only independent risk factor related to lymph node metastasis, which suggests that preoperative evaluation of the depth of duodenal invasion is more sensitive for predicting nodal involvement than gross appearance. In the current study, T1 tumors were subdivided into layer I and layer II, and no lymph node metastasis was found when tumors were confined to layer I (the mucosa). When primary carcinoma invaded layer II (the submucosa), the nodal metastasis rate increased up to $25 \%$, in agreement with some studies reporting that tumor invasion limited to the mucosa did not have nodal spread, and an increased risk (20-28\%) of lymph node metastasis was found in CPV invading the sphincter of Oddi $(10,11,23)$. Consequently, accurate preoperative differential diagnosis of layer I and layer II invasion is imperative, and only patients with duodenal invasion limited to layer I are suitable for local resection. Otherwise, PD with nodal clearance should be the preferred choice. 


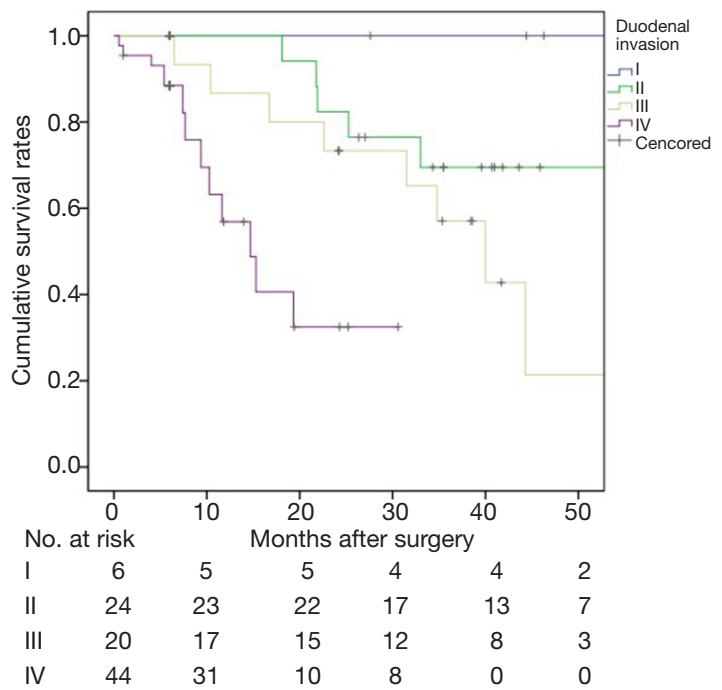

Figure 3 Kaplan-Meier survival curves for patients with stratifications of duodenal invasion after resection for carcinoma of the papilla of Vater. There was a clinically important difference in survival among the groups (log-rank test, $\mathrm{P}<0.001$ ). Moreover, all curves were independent of each other in the figure.

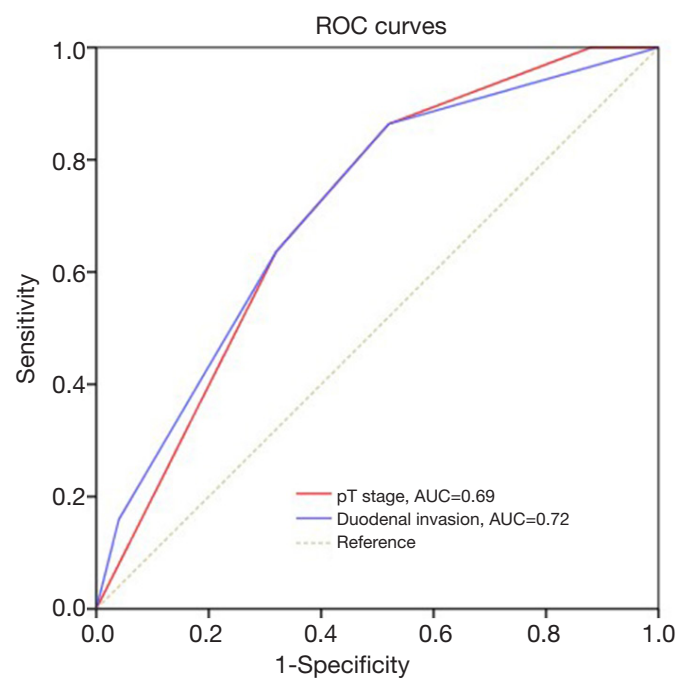

Figure 4 Receiver operating characteristic (ROC) curves for two models to predict survival. Area under the ROC curve (AUC) value indicates that the prediction accuracy of the model based on duodenal invasion is better than the one based on pT stage.

The diagnostic accuracy of stage T1 CPV was reported to be $73-93 \%$ by EUS (24-26). However, EUS cannot accurately visualize the sphincter of Oddi because of its hyper-echo image $(10,27,28)$. In fact, sphincter of
Oddi invasion was identified in four patients, and one of them $(25 \%)$ showed lymph node positivity in our study. Consequently, EUS has been regarded as insufficient in preoperative diagnosis of the depth of duodenal invasion at present. Based on this, our duodenal classification system incorporates the sphincter of Oddi into layer II (the submucosa), which can theoretically further improve the diagnostic accuracy of duodenal stratification between layer I and layer II invasion by EUS. However, the results still need to be further explored. At present, only IDUS can depict the sphincter of Oddi as a hypoechoic layer. IDUS has been reported to be superior to EUS in terms of diagnostic accuracy ( $88.9 \%$ vs. $56.3 \%)$, and the diagnostic accuracy for stage T1 CPV is $80-100 \%(24,27,29)$. Thus, it is possible and feasible to completely discriminate between layer I and layer II invasion in stage T1 by IDUS and then to select patients suitable for local resection.

The 5-year survival rate ranges from $30 \%$ to $67 \%$ in patients with CPV after PD (30-32). In the present study, the 5 -year overall survival was $45.2 \%$ (Figure 1). Various prognostic factors in patients with CPV after curative resection have been reported, including gross appearance, depth of tumor infiltration (pT stage), pancreatic invasion, perineural invasion, venous invasion and nodal involvement $(4,6,12,15,31-33)$. Our results are consistent with previous research and seem to indicate that the depth of duodenal invasion and nodal involvement are the most important factors affecting long-term survival because venous invasion is extremely rare in stage T1 CPV (Table 2). Moreover, previous analysis concluded that the depth of duodenal invasion was the only independent risk factor associated with nodal involvement. Therefore, an accurate preoperative evaluation of the depth of duodenal invasion is imperative to predict long-term survival so as to guide the choice of optimal surgical procedure or adjuvant treatment in patients with stage T1 CPV.

The depth of duodenal invasion is an important component of the $\mathrm{T}$ stage in $\mathrm{CPV}$, and its role in predicting prognosis has been increasingly valued. According to new update of the $8^{\text {th }}$ edition of the AJCC staging system, the previous $\mathrm{T} 2$ was expanded with more precise definitions based on the depth of duodenal invasion (16). It can be seen from Figure 2 that the new subclassification of T2 and $\mathrm{T} 3$ was unreasonable because no prognostic difference was found $(\mathrm{P}=0.073)$. However, the separate categorization of $\mathrm{T} 1$ and $\mathrm{T} 4$ was justified. Based on our duodenal stratification, all layers, especially layer III (equivalent to T2) and layer IV, were independent of each other and showed 
better prognostic differences (Figure 3). ROC test further verified the conclusion (Figure 4). Multivariate analysis also demonstrated that the detailed depth of duodenal invasion was a stronger predictor of prognosis than the new $T$ staging. Therefore, the classification of duodenal invasion proposed in the current study is more suitable for providing accurate predictions on the prognosis of $\mathrm{CPV}$ than the new $\mathrm{T}$ category. A modified $\mathrm{T}$ category needs to be proposed based on the detailed depth of duodenal invasion. In particular, T3 should be distinguished by the serosa layer (layer IV), which is in agreement with a recent study (33). Interestingly, our study found that perineural invasion in CPV had strong prognostic significance and was correlated with nodal involvement, which suggests that perineural dissection during surgery is crucial to ensure R0 resection in order to improve long-term survival (32).

However, this study has certain limitations: this was a retrospective, single-center study with a limited sample size; only six patients were classified with layer I duodenal invasion. There are two possible reasons. First, when patients developed clinical symptoms, tumor invasion had often progressed. Second, some select patients who were not included might have chosen to undergo local resection (perhaps they were believed to have "early tumors"). In addition, follow-up time was not long enough. Accordingly, we are not yet able to draw final conclusions. Additional nodal involvement data are needed from patients with tumor invasion limited to the mucosa.

In conclusion, the depth of duodenal invasion is a strong predictor of nodal involvement and prognosis in CPV. An accurate preoperative evaluation of the depth of duodenal invasion can be implemented by IDUS. PD with lymphadenectomy should be the first treatment option in stage T1 CPV, only patients with duodenal invasion limited to the mucosa have no nodal involvement and are appropriate for local resection. The stratification of duodenal invasion proposed in the present study is applicable for providing additional precise information regarding the prognosis than the new $\mathrm{T}$ staging system. A modified $T$ category needs to be proposed based on the detailed depth of duodenal invasion.

\section{Acknowledgments}

We thank American Journal Experts (AJE) for writing assistance with an earlier version of the manuscript.

Funding: This work was supported by CAMS Innovation Fund for Medical Sciences (CIFMS) (No. 2016-I2M-1-001).

\section{Footnote}

Reporting Checklist: The authors have completed the STROBE reporting checklist. Available at http://dx.doi. org/10.21037/tcr-20-1914

Data Sharing Statement: Available at http://dx.doi. org/10.21037/tcr-20-1914

Peer Review File: Available at http://dx.doi.org/10.21037/tcr20-1914

Conflicts of Interest: All authors have completed the ICMJE uniform disclosure form (available at http://dx.doi. org/10.21037/tcr-20-1914). The authors have no conflicts of interest to declare.

Ethical Statement: The authors are accountable for all aspects of the work in ensuring that questions related to the accuracy or integrity of any part of the work are appropriately investigated and resolved. The study was conducted in accordance with the Declaration of Helsinki (as revised in 2013). The study was reviewed and approved by the institutional review board of Eastern Hepatobiliary Surgery Hospital (EHBHKY2019-003-038), Shanghai, China. Written informed consent was obtained from all patients.

Open Access Statement: This is an Open Access article distributed in accordance with the Creative Commons Attribution-NonCommercial-NoDerivs 4.0 International License (CC BY-NC-ND 4.0), which permits the noncommercial replication and distribution of the article with the strict proviso that no changes or edits are made and the original work is properly cited (including links to both the formal publication through the relevant DOI and the license). See: https://creativecommons.org/licenses/by-nc-nd/4.0/.

\section{References}

1. Albores-Saavedra J, Schwartz AM, Batich K, et al. Cancers of the ampulla of vater: demographics, morphology, and survival based on 5,625 cases from the SEER program. J Surg Oncol 2009;100:598-605.

2. Kayahara M, Nagakawa T, Ohta T, et al. Surgical strategy for carcinoma of the papilla of Vater on the basis of lymphatic spread and mode of recurrence. Surgery 1997;121:611-7. 
3. Beger HG, Thorab FC, Liu Z, et al. Pathogenesis and treatment of neoplastic diseases of the papilla of Vater: Kausch-Whipple procedure with lymph node dissection in cancer of the papilla of Vater. J Hepatobiliary Pancreat Surg 2004;11:232-8.

4. Moriya T, Kimura W, Hirai I, et al. Nodal involvement as an indicator of postoperative liver metastasis in carcinoma of the papilla of Vater. J Hepatobiliary Pancreat Surg 2006;13:549-55.

5. Bogoevski D, Chayeb H, Cataldegirmen G, et al. Nodal microinvolvement in patients with carcinoma of the papilla of vater receiving no adjuvant chemotherapy. J Gastrointest Surg 2008;12:1830-7; discussion 1837-8.

6. Todoroki T, Koike N, Morishita Y, et al. Patterns and predictors of failure after curative resections of carcinoma of the ampulla of Vater. Ann Surg Oncol 2003;10:1176-83.

7. Winter JM, Cameron JL, Olino K, et al. Clinicopathologic analysis of ampullary neoplasms in 450 patients: implications for surgical strategy and long-term prognosis. J Gastrointest Surg 2010;14:379-87.

8. Kobayashi A, Konishi M, Nakagohri T, et al. Therapeutic approach to tumors of the ampulla of Vater. Am J Surg 2006;192:161-4.

9. Yoon SM, Kim MH, Kim MJ, et al. Focal early stage cancer in ampullary adenoma: surgery or endoscopic papillectomy? Gastrointest Endosc 2007;66:701-7.

10. Aiura K, Hibi T, Fujisaki H, et al. Proposed indications for limited resection of early ampulla of Vater carcinoma: clinico-histopathological criteria to confirm cure. J Hepatobiliary Pancreat Sci 2012;19:707-16.

11. Lee H, Park JY, Kwon W, et al. Transduodenal Ampullectomy for the Treatment of Early-Stage Ampulla of Vater Cancer. World J Surg 2016;40:967-73.

12. Kayahara M, Ohta T. Gross appearance of the ampullary tumor predicts lymph node metastasis and outcome. Dig Surg 2010;27:127-31.

13. Terasawa H, Uchiyama K, Tani M, et al. Impact of lymph node metastasis on survival in patients with pathological T1 carcinoma of the ampulla of Vater. J Gastrointest Surg 2006;10:823-8.

14. Sikora SS, Balachandran P, Dimri K, et al. Adjuvant chemo-radiotherapy in ampullary cancers. Eur J Surg Oncol 2005;31:158-63.

15. Qiao QL, Zhao YG, Ye ML, et al. Carcinoma of the ampulla of Vater: factors influencing long-term survival of 127 patients with resection. World J Surg 2007;31:137-43; discussion 144-6.

16. Chun YS, Pawlik TM, Vauthey JN. 8th Edition of the
AJCC Cancer Staging Manual: Pancreas and Hepatobiliary Cancers. Ann Surg Oncol 2018;25:845-7.

17. Miyazaki M, Ohtsuka M, Miyakawa S, et al. Classification of biliary tract cancers established by the Japanese Society of Hepato-Biliary-Pancreatic Surgery: 3(rd) English edition. J Hepatobiliary Pancreat Sci 2015;22:181-96.

18. Horiguchi S, Kamisawa T. Major duodenal papilla and its normal anatomy. Dig Surg 2010;27:90-3.

19. Morozumi A, Fujino MA, Sato T, et al. Endosonographic criteria for assessment of the depth of duodenal invasion in carcinoma of the papilla of Vater. Digestive Endoscopy 2001;13:149-58.

20. Jin $G$, Sugiyama $M$, Tuo $H$, et al. Distribution of lymphatic vessels in the neural plexuses surrounding the superior mesenteric artery. Pancreas 2006;32:62-6.

21. Falconi M, Crippa S, Dominguez I, et al. Prognostic relevance of lymph node ratio and number of resected nodes after curative resection of ampulla of Vater carcinoma. Ann Surg Oncol 2008;15:3178-86.

22. Chen CH, Tseng LJ, Yang CC, et al. Preoperative evaluation of periampullary tumors by endoscopic sonography, transabdominal sonography, and computed tomography. J Clin Ultrasound 2001;29:313-21.

23. Amini A, Miura JT, Jayakrishnan TT, et al. Is local resection adequate for T1 stage ampullary cancer? HPB (Oxford) 2015;17:66-71.

24. Ito K, Fujita N, Noda Y, et al. Preoperative evaluation of ampullary neoplasm with EUS and transpapillary intraductal US: a prospective and histopathologically controlled study. Gastrointest Endosc 2007;66:740-7.

25. Trikudanathan G, Njei B, Attam R, et al. Staging accuracy of ampullary tumors by endoscopic ultrasound: meta-analysis and systematic review. Dig Endosc 2014;26:617-26.

26. Peng CY, Lv Y, Shen SS, et al. The impact of endoscopic ultrasound in preoperative evaluation for ampullary adenomas. J Dig Dis 2019;20:248-55.

27. Itoh A, Goto H, Naitoh $\mathrm{Y}$, et al. Intraductal ultrasonography in diagnosing tumor extension of cancer of the papilla of Vater. Gastrointest Endosc 1997;45:251-60.

28. Ridtitid W, Schmidt SE, Al-Haddad MA, et al. Performance characteristics of EUS for locoregional evaluation of ampullary lesions. Gastrointest Endosc 2015;81:380-8.

29. Fujita N, Noda Y, Kobayashi G, et al. Intraductal ultrasonography (IDUS) for the diagnosis of biliopancreatic diseases. Best Pract Res Clin Gastroenterol 
2009;23:729-42.

30. Duffy JP, Hines OJ, Liu JH, et al. Improved survival for adenocarcinoma of the ampulla of Vater: fiftyfive consecutive resections. Arch Surg 2003;138:941-8; discussion 948-50.

31. Kim RD, Kundhal PS, McGilvray ID, et al. Predictors of failure after pancreaticoduodenectomy for ampullary carcinoma. J Am Coll Surg 2006;202:112-9.

32. Sudo T, Murakami Y, Uemura K, et al. Prognostic impact

Cite this article as: $\mathrm{Gu}$ Z, Li Z, Yu W, Zhang Y, Wang C. Choice of surgical procedures for patients with stage T1 carcinoma of the papilla of Vater: a retrospective study. Transl Cancer Res 2020;9(11):7113-7124. doi: 10.21037/tcr-20-1914 of perineural invasion following pancreatoduodenectomy with lymphadenectomy for ampullary carcinoma. Dig Dis Sci 2008;53:2281-6.

33. Imamura T, Yamamoto Y, Sugiura T, et al. The Prognostic Relevance of the New 8th Edition of the Union for International Cancer Control Classification of TNM Staging for Ampulla of Vater Carcinoma. Ann Surg Oncol 2019;26:1639-48. 